inverse to their size, the theory of restricted diffusion through pores of fixed size ${ }^{1}$ was attractive at first sight. However, the failure of electron microscopy to reveal such pores threw the theorists into confusion. Small molecules probably pass through the endothelial cytoplasm by way of general cell transport mechanisms. The situation with regard to larger molecules is very uncertain. Two possibilities seem to exist ; carriage within intracytoplasmic vesicles (caveolae intracellularis) or passage along tight interendothelial junctions, which may relax imperceptibly to allow transfer. One of the best recent experiments quoted by Florey suggests that both mechanisms operate.

In inflammation the permeability of vascular endothelial walls increases enormously so that large amounts of plasma protein escape to form the characteristic protein-rich exudate. ${ }^{2}$ The now classical paper by G. Majno and G. E. Palade ${ }^{3}$ showed conclusively that increased vascular permeability, induced for example by histamine, was due to a short-lived opening up of the normally tight interendothelial junctions. The existence of these gaps in inflammation itself has been shown by R. S. Cotran ${ }^{4}$ and Kathryn N. Ham and J. V. Hurley.5 Junction-opening after application of histamine or bradykinin is largely confined to venules with little apparent effect on true capillaries. ${ }^{3}$ The same may be true at least of some types of injury, ${ }^{6}$ but the relative contribution of venules and capillaries to inflammatory exudates is uncertain and may well be variable. ${ }^{i}$

It is now known, also from electron microscopy, that the leucocytes that enter the tissues in inflammation do so via the interendothelial junctions by inserting pseudopodia into the potential space. ${ }^{8}$ This process may continue in the absence of plasma protein leakage from the affected vessel. ${ }^{6}$ One possible exception to the intercellular route is the intracytoplasmic transport of small lymphocytes reported to occur in normal lymph-node venules."

Another pathological situation in which the vascular endothelium is important is atherosclerosis. This problem may prove to be specially related to the question of the transport of lipid macromolecules across the vessel wall ${ }^{10}$ and is clearly a promising field for research.

As pointed out in Majno's outstanding review, ${ }^{11}$ one of the major issues to be resolved is the discrepancy between the electron microscopic and physiological data concerning the permeability of endothelial walls. When, however, this gap " of only a few Angstroms" $"$ is bridged, the basic problem of the mechanism of junction opening and leucocytic diapedesis after injury will still remain.

\section{Mammography as a Screening Test for Breast Cancer}

Breast cancer in women is the cause of approximately onequarter of cancer deaths and of about three in every 100 deaths from all causes. In spite of improvements in treatment and new methods of palliation there is no evidence that the death rate is decreasing. All experience suggests that the earlier treatment is established the better is the chance of a permanent cure. Many methods have been advocated to encourage early detection; these include selfexamination by the patient, routine clinical examination at special clinics, and recently the use of $x$-ray mammography. ${ }^{1}$
This is a technique for soft-tissue radiography of the breast and can detect malignant tumours which are impalpable on clinical examination.

In December 1963 the Health Insurance Plan of Greater New York, in cooperation with certain medical groups, launched a screening programme for breast cancer by both mammography and clinical examination; and recently $S$. Shapiro, P. Strax, and L. Venet ${ }^{2}$ reported on the first two years' experience of this experiment. Women between the ages of 40 and 64 years taking part in the Health Insurance Plan were randomly allocated to either a control or a study group. Those in the study group were offered a screening examination at their medical centre and encouraged to attend for two similar annual follow-up examinations. The women in the control group followed their usual practice for medical care and no special effort was made to encourage them to be examined. So far, 9,883 examinations have been carried out on the study group, and, either on clinical or on radiological grounds, 227 women have been recommended for a biopsy or an aspiration. Of these, 145 agreed to an operation, and 23 cancers were confirmed by pathological examination. At subsequent mastectomy $16(70 \%)$ of these had no evidence of malignant deposits in the regional lymph nodes. Four further cases of cancer were reported between screening examinations, making a total of 27 cases for the study group. In the control group during a similar period 14 cases of breast cancer occurred, of which eight $(57 \%)$ had no evidence of spread of the disease.

No conclusions are drawn from these preliminary data, and prolonged follow-up, including mortality figures, will be needed for a useful assessment of the results. However, it may be prudent at this stage to consider the value and practicality of mammography as a screening procedure if it were applied to the population in general. Are we likely to see the technique introduced within the next few years and become as important in the detection of breast cancer as the smear for cervical cancer?

There would certainly be considerable difficulties in introducing such a scheme. In the study reported by Shapiro and his colleagues only 13 patients could be examined both clinically and radiologically in a three-hour session. A programme for the general population would create practical and economic problems on a scale that would put it beyond the resources of most countries. In addition to the time and the skilled medical assistance needed for such a service, provision would have to be made for carrying out biopsy procedures on approximately $2 \%$ of those women who were examined. Furthermore, the optimum time for re-examination is not yet known, though it seems likely that the procedures would have to be repeated annually.

For these techniques to be of value some means must be found to recognize a group of women who run an abnormally high risk of developing breast cancer. These women could then be studied at special centres properly equipped for this purpose, and if any tumour was detected either clinically or radiologically then the appropriate steps could be taken. So far no methods exist for identifying such a highrisk group. Though the incidence of breast cancer is

\footnotetext{
1 Stevens, G. M., and Weigen, J. F., Cancer (Philad.), 1966, 19, 51. Shapiro, S., Strax, P., and Venet, L., f. Amer. med. Ass., 1966, 195, 731.

s Bilbrook, R. D., Hayward, J. L., Spicer, C. C., and Thomas, B. S., Lancet, 1962, 2, 1238

4 Hayward, J. L., Brit. F. Surg., 1964, 51, 224

- Brit. med. F., 1965, 2, 1198. L. J., Lancet, 1966, 1690.
} 
increased in certain types of women, such as those without children and those with a family history of the disease, none of the differences is great enough for it to be of practical use. However, recent work $^{3}$ has shown that many women with early breast cancer excrete abnormal amounts of steroids in their urine, and a trial is in progress ${ }^{45}$ to investigate whether this abnormality precedes the onset of the disease. It has also been reported ${ }^{6}$ that buccal smears from many patients with breast cancer show certain sexchromatin anomalies in the epithelium which are not present in normal women or patients with other malignant tumours. If either or both of these findings could be used to formulate a high-risk group then we might see mammography and clinical examination being used with advantage to detect the early disease. Under such circumstances we might at last look forward to an overall improvement in the results of treatment.

\section{Closed Abdominal Trauma}

Attention was drawn recently ${ }^{1}$ to the rise in the frequency of stab wounds of the abdomen. Injuries due to blunt abdominal trauma are also becoming increasingly common, mainly as a result of the rising numbers of road traffic accidents.

Closed injuries of the abdomen present a challenging problem in management, as prompt diagnosis and treatment are essential if lives are to be saved. Not only is the patient usually severely shocked, with damage to more than one intraabdominal viscus, but frequently there are also associated injuries elsewhere. R. D. Williams and A. A. Yurko recently reviewed 278 consecutive patients admitted to the Ohio State University Hospital in Columbus as a result of blunt abdominal trauma. Almost two-thirds of the injuries were due to road accidents. By far the commonest organs injured were the kidney and the spleen, followed in order by the liver, the pancreas, the bladder, and the intestines. More than one intra-abdominal organ had been injured in 84 patients, and in addition one-third had injuries to the chest, one-third injuries to the head, and one-quarter damage to the arms or legs. These more obvious injuries often distract attention away from the abdomen and cause delay in treatment and an increase in mortality, either because of the delay or because of the severity of the injuries themselves. Indeed, H. Ellis, P. W. W. Griffiths, and A. Macintyre ${ }^{3}$ found that of the nine deaths which occurred in 30 patients with traumatic haemoperitoneum from ruptures of the spleen or liver or both, eight had severe associated head injuries, and the ninth had multiple injuries elsewhere.

The diagnosis that an intra-abdominal viscus has been torn is largely a clinical one, and the laboratory can provide only slight help to the surgeon. Williams and Yurko found that $x$-ray examination of the abdomen was of relatively little value, and in only $11 \%$ of their cases were the films helpful. An $x$-ray film of the chest is useful, however, in excluding an

1 Brit. med. F., 1966, 1, 1437.

-Williams, R. D., and Yurko, A. A., Amer. f. Surg., 1966, 111, 477.

- Ellis, H., Griffiths, P. W. W., and Macintyre, A., Brit. f. Surg., 1958, 45, 606.

- Nick, W. V., Zollinger, R. W., and Williams, R. D., f. Trauma, 1965, $5,495$.

s Ellis, H., and Mynors, J. M., Postgrad. med. F., 1959, 35, 683.

- McIndoe, A. H., Brit. F. Surg., 1932, 20, 249.

, Sizer, J. S., Wayne, E. R., and Frederick, P. L., Arch. Surg., 1966, 92, 362. associated injury to the lungs or the diaphragm. Elevation of the white cell count may reflect the degree of general trauma but has no specific diagnostic value. W. V. Nick, R. W. Zollinger, and R. D. Williams ${ }^{4}$ stress the value of estimation of the serum amylase in the diagnosis of injuries of the pancreas, though the level is not always raised in such cases. ${ }^{5}$ The presence of haematuria is valuable evidence of injury to the genito-urinary tract, and the urine must be tested in every case of abdominal trauma, no matter how trivial. When there is doubt whether an intra-abdominal organ has been damaged needle paracentesis may be useful. Williams and Yurko found that this technique gave the right answer in $90 \%$ of cases, but they stress that failure to aspirate blood does not exclude the existence of injury.

A further complication is the possibility of delayed rupture of viscera which may occur after blunt injury; this applies particularly to the spleen, as was first stressed by $\mathrm{Sir}$ Archibald McIndoe. ${ }^{6}$ J. S. Sizer, E. R. Wayne, and P. L. Frederick $^{7}$ have reviewed 312 examples of delayed splenic rupture. Half of these occurred between the second and seventh days after the injury, $25 \%$ after the first week, and $10 \%$ of the cases were delayed for more than one month. Apparently what happens is that a subcapsular splenic haematoma ruptures, and this is often precipitated by coughing or sneezing. The mortality in such cases is in the region of $10 \%$ and correct diagnosis was made in only $20 \%$. Delayed rupture of the damaged liver and bowel may also occur.

Patients with closed and isolated injuries to the kidneys can usually be managed conservatively, though occasionally bleeding will continue, when urgent exploration and probably nephrectomy will be required. If there is any suspicion of damage to the other viscera laparotomy is imperative. In such cases a midline incision is best as it allows rapid entry, easy extension in an upward or downward direction, and the ready exposure of all the intra-abdominal organs. Ease of exposure is specially important, since it is always possible that more than one viscus will be found to be damaged-particularly when the injuries result from a road accident.

\section{Liver Necrosis from Paracetamol}

Most people regard paracetamol as a useful if somewhat expensive alternative to aspirin, especially when the latter causes gastro-intestinal symptoms. It is a relatively mild analgesic and in therapeutic doses appears to be free from side-effects. No evidence of nephrotoxicity was found when paracetamol was administered to healthy volunteers, ${ }^{1}$ but the fact that it is a breakdown product of phenacetin should serve as a warning that prolonged administration might lead to renal damage. Idiosyncrasy to the drug probably explains rare cases of thrombocytopenic purpura and agranulocytosis, ${ }^{2}$ and the occasional patient who is sensitive to aspirin may also be unable to tolerate paracetamol.

This week we puhlish two papers reporting the serious consequences of overdosage with paracetamol. At page 497 Drs. D. G. D. Davidson and W. N. Eastham describe two patients who died within four days of taking $25 \mathrm{~g}$. and $75 \mathrm{~g}$. respectively of the drug. The first had clinical signs of

\footnotetext{
1 Prescott, L. F., Lancet, 1965, 2, 91.

2 Lloyd, T. W., ibid., 1961, 1, 114.
} 